\title{
Lipiodol Uptake and Wash-out Rate Detection on Computed Tomography as Predictors for Tumor Recurrence in Hepatocellular Carcinoma Treated by Transcatheter Arterial Chemoembolization
}

\author{
Andreea-Elena Scheau ${ }^{1}$, Cristian Scheau ${ }^{2}$, Ioana Gabriela Lupescu ${ }^{1,2}$
}

Corresponding author:

Andreea-Elena Scheau, MD, PhD student

Department of Radiology, Medical

Imaging and Interventional Radiology,

Fundeni Clinical Institute, Bucharest,

Romania, 258 Fundeni Street

E-mail: andreea.ghergus@gmail.com
'Department of Radiology, Medical Imaging and Interventional Radiology, Fundeni Clinical Institute

"Carol Davila" University of Medicine and Pharmacy, Bucharest, Romania

2"Carol Davila" University of Medicine and Pharmacy, Bucharest, Romania

\section{ABSTRACT}

Background: Transcatheter arterial chemoembolization (TACE) is a dedicated method for treating intermediate stage hepatocellular carcinoma (HCC). The Lipiodol used in conventional TACE presents the advantage of being easily visualized and quantified on Computed Tomography (CT).

Methods: Immediate postprocedural (baseline) and follow-up CT examinations of twenty patients treated by TACE for HCC were evaluated in this study. Lipiodol uptake on baseline images and progressive washout on follow-up examinations were assessed.

Results: Statistically significant lower baseline Lipiodol densities are found in patients that develop tumoral recurrence at some point on follow-up examinations $(p=0.0280)$. Using the treated nodule density as a predictive factor for tumoral recurrence, we obtained a diagnostic sensitivity of $92.31 \%$ in patients with cut-off values $\leq 545.36 \mathrm{HU}$, albeit with a low specificity. All patients develop a quantifiable Lipiodol density decrease over time, regardless of tumoral recurrence implication $(p=0.0298)$. There is evidence to suggest that specific Lipiodol accumulation patterns may be associated with a higher risk of tumor recurrence.

Conclusions: Baseline post-procedural Lipiodol accumulation levels may serve as a predictor for tumoral recurrence in patients treated for HCC using TACE, with good sensitivity provided a high cut-off value is set for the measured densities.

Key words: transcatheter arterial chemoembolization, hepatocellular carcinoma, Lipiodol, wash-out, surveillance, tumoral recurrence.

\section{INTRODUCTION}

Hepatocellular carcinoma $(\mathrm{HCC})$ is the most frequent primary malignant tumor of the liver, responsible for up to one million deaths, globally, per year (1). While most cancers worldwide are experiencing a drop in incidence, the burden of HCC is increasing, with a heterogeneous distribution among the population and with challenges concerning therapeutic options (2). One of the treatment modalities for $\mathrm{HCC}$ is transcatheter arterial chemoembolization (TACE), which is appropriate for stage B HCC according to Barcelona Clinic Liver Cancer staging system (3). TACE experienced many technical developments as new drugs 
and delivery systems were developed, but the conventional approach, using Lipiodol emulsion mixed with doxorubicin followed by complete embolization is still one of the most used methods worldwide (4). The follow-up of TACE-treated HCC is performed by computed tomography (CT) and/or magnetic resonance imaging (MRI). Certain imaging criteria have been developed to assess the success rate of treatment and the appearance of tumoral recurrence (5). Taking in consideration the chemotherapeutic drug is mixed with Lipiodol, and the latter is easily visualized on CT (and less so on MRI), the degree of Lipiodol uptake and potential subsequent wash-out from the tumor nodule may provide information regarding the effectiveness of the procedure (6). This paper evaluates the dynamics of Lipiodol in HCC patients treated by TACE on follow-up studies, attempting to verify the potential of using Lipiodol uptake and wash-out rate as a predictor for tumor recurrence.

\section{METHODS}

The retrospective study group consisted of all patients $(n=20)$ treated for HCC with conventional TACE between January 2014 and December 2014 in the Department of Radiology, Medical Imaging and Interventional Radiology of Fundeni Clinical Institute, Bucharest. The patients were submitted to baseline and follow-up imaging studies in our center, and they were tracked up until August 2017.

Inclusion criteria were set as followed: patients with both pre-interventional and follow-up CT scans in our Department, using dedicated protocols; treatment criteria according to HCC staging were respected; patients treated with conventional TACE were selected; tumor's smallest diameter larger than $10 \mathrm{~mm}$; HCC was confirmed with either biopsy or certainty of imaging features; patients developed HCC on a chronic liver disease.

For each patient included, if multiple nodules were present, only one nodule was considered for the study, which was the largest and showed dominant features.

Measured parameters were patient age, gender, etiology of chronic liver disease as well as tumor size, Lipiodol uptake size, shape and mean density in both baseline and follow-up imaging studies. Appearance of tumoral recurrence was diagnosed using mRECIST criteria (7). Lipiodol uptake density was measured in Hounsfield units (HU) using a round region of interest (ROI) within the largest diameter of the tumor, on the unenhanced series of the study. Lipiodol uptake was classified as either being complete, heterogeneous, peripheral, nodular or absent. Lipiodol wash-out was defined as either partial or complete decrease in density in the treated nodule. The degree of Lipiodol washout in relation to time was measured as the difference between follow-up and baseline studies' densities divided by the time period expressed in months. Patient follow-up was terminated on the appearance of tumoral recurrence, employment of subsequent nodule treatment, complete wash-out, patient withdrawal or death.

The images in the studies were assessed in consensus by three board certified radiologists. Images were interpreted on a dedicated imaging software OsiriX 8.0.2 MD 64bit and all data was collected using Microsoft Excel 2013 15.0.4953.1000 64-bit.

Statistical data was analyzed using IBM SPSS Statistics 21 Premium x64bit. Standard statistical methods like mean, median, average and standard deviation were employed. Receiver operating characteristic (ROC) curves were used to determine predictive power. Mann-Whitney $U$ test was employed to test statistical differences between patient subgroups. Pearson correlation coefficient was used to measure correlation power between variables. A $p$-value smaller than 0.05 was considered statistically significant.

Hospital ethics committee approval and patient informed consent were obtained.

\section{RESULTS}

A total of 20 patients were included in this study $(\mathrm{M}: \mathrm{F}=11: 9)$ aged between 23 and 85 years (median age: 68 years old). All patients had been diagnosed with $\mathrm{HCC}$ on liver cirrhosis. The underlying cause of cirrhosis consisted of: hepatitis $C$ virus $(n=11)$, hepatitis B virus $(n=5$, out of which one was antigen delta positive as well), alcoholic ( $n=2)$ and cryptogenic $(n=2)$.

Out of the total number of patients included in the study, 13 patients (65\%) demonstrated viable tumoral tissue on the follow-up examinations.

Histopathological evidence of hepatocellular carcinoma was available in 6 cases (30\%).

Initial tumoral nodule size ranged between 10.36 $\mathrm{mm}$ and $84.06 \mathrm{~mm}$, with an average of $32.57 \mathrm{~mm}$ $( \pm 21.23 \mathrm{~mm}$ ). First follow-up examination time period ranged from 1 to 13 months (median of 3 months). The mean time for tumoral recurrence identification is $4.3 \pm$ 2.1 months.

The mean density of baseline Lipiodol uptake was $341.3 \pm 268.1 \mathrm{HU}$, and for the follow-up studies it averaged $239.8 \pm 192.7 \mathrm{HU}$.

Tumor recurrence occurred in 1 out of 4 cases with complete Lipiodol uptake, in 9 out of 11 heterogeneous 
uptake patterns, 1 out of 2 nodular patterns and 2 out of 2 cases of absent uptake. Ring-shaped uptake pattern demonstrated no tumoral recurrence up until the last follow-up examination available. There is no statistical correlation between Lipiodol accumulation patterns and tumoral recurrence appearance $(p=$ 0.1415).

Lipiodol uptake values at baseline, in patients with no tumor recurrence in the follow-up period, were $437.6 \pm 188.6 \mathrm{HU}$, and dropped to $300.1 \pm 173.4 \mathrm{HU}$ for the entire follow-up period. In patients with tumor recurrence, the baseline Lipiodol uptake values were $289.4 \pm 289.2 \mathrm{HU}$ and decreased to $190.1 \pm 193.7 \mathrm{HU}$ in subsequent post-interventional studies (fig. 1).

The difference between baseline Lipiodol uptake in patients with or without tumor recurrence is statistically significant when eliminating outliers $(p=0.0280)$. Also, a statistically significant difference is noted between Lipiodol mean densities at the follow-up examinations between the two subgroups, with or without tumoral recurrence $(p=0.0126)$. There was an overall Lipiodol density decrease over time in all patients, regardless of the presence of the viable tumor tissue presence $(p=0.0298)$.

A ROC curve has been calculated in order to estimate baseline Lipiodol densities' predictive power in the diagnosis of tumoral recurrence (table 1). Using the baseline CT as a predictor, a cut-off value of $\leq 390.03 \mathrm{HU}$ provides a sensitivity of $84.62 \%$ and a specificity of $57.14 \%(p=0.0294)$.

\section{DISCUSSION}

The low percentage of histological evidence in the

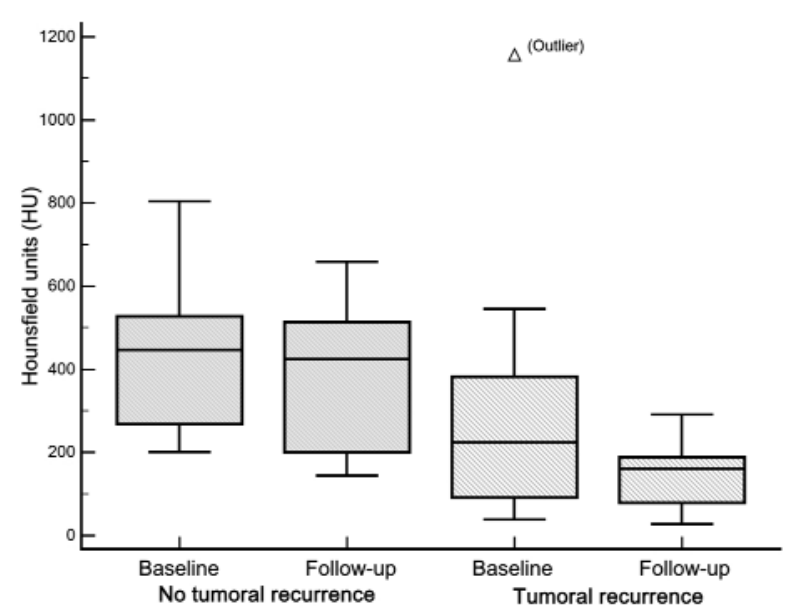

Figure 1 - Mean Lipiodol densities in patients with or without tumoral recurrence, at baseline and follow-up examinations. 95\% confidence intervals displayed as error bars. Outlier which was excluded in calculations is identified in baseline tumoral recurrence subgroup.

treated patients in our group of patients is coherent with recent clinical practice, which renders biopsy no longer a requirement for the diagnosis of HCC. The rationale for this strategy is that imaging criteria is considered sufficient in most cases (8).

The large interval of variation for the first follow-up examination after the TACE treatment is usually explained by low patient compliance, which is an important factor in overall adhesion to treatment and surveillance (9). The small size of our patient group does not allow for a significant study of the correlation between etiology of liver disease and compliance. However, literature seems to suggest low adherence in patients with chronic hepatitis, which may be a factor in

Table 1 - ROC curve criterion values and coordinates used to predict sensitivity and specificity of using baseline Lipiodol as a predictor for tumoral recurrence. The chosen cut-off value in the first column offers a corresponding sensitivity and specificity, with according $95 \%$ confidence intervals. $\mathrm{HU}=$ Hounsfield units. $\mathrm{Cl}=$ Confidence interval.

\begin{tabular}{|c|c|c|c|c|}
\hline Criterion (HU) & Sensitivity & $95 \% \mathrm{Cl}$ & Specificity & $95 \% \mathrm{Cl}$ \\
\hline$<37.23$ & 0 & $0.0-24.7$ & 100 & $59.0-100.0$ \\
\hline$\leq 190.09$ & 46.15 & $19.2-74.9$ & 100 & $59.0-100.0$ \\
\hline$\leq 200.16$ & 46.15 & $19.2-74.9$ & 85.71 & $42.1-99.6$ \\
\hline$\leq 224.92$ & 53.85 & $25.1-80.8$ & 85.71 & $42.1-99.6$ \\
\hline$\leq 234.25$ & 53.85 & $25.1-80.8$ & 71.43 & $29.0-96.3$ \\
\hline$\leq 245.49$ & 69.23 & $38.6-90.9$ & 71.43 & $29.0-96.3$ \\
\hline$\leq 369.09$ & 69.23 & $38.6-90.9$ & 57.14 & $18.4-90.1$ \\
\hline$\leq 390.03$ & 84.62 & $54.6-98.1$ & 57.14 & $18.4-90.1$ \\
\hline$\leq 461.39$ & 84.62 & $54.6-98.1$ & 28.57 & $3.7-71.0$ \\
\hline$\leq 545.36$ & 92.31 & $64.0-99.8$ & 28.57 & $3.7-71.0$ \\
\hline$\leq 803.2$ & 92.31 & $64.0-99.8$ & 0 & $0.0-41.0$ \\
\hline$\leq 1156.85$ & 100 & $75.3-100.0$ & 0 & $0.0-41.0$ \\
\hline
\end{tabular}


long delays of follow-up imaging studies (10). As such, calculated mean time to the appearance of tumoral recurrence may be considerably decreased by shorter times to follow-up in patients treated for HCC (11).

No statistically significant correlation was found between Lipiodol accumulation patterns and the appearance of tumoral recurrence in our study (fig. 2), regardless of nodule size. Nevertheless, other study shows that complete retention patterns have a higher chance for a complete response in comparison to than patients with moderate or poor retention patterns (12).

A quantifiable progressive Lipiodol wash-out was identified in all patients, regardless of whether they eventually developed a tumoral recurrence or not. A decrease in size, as well as a steady drop of mean densities in treated nodules were commonly found in patients without tumoral recurrence, and was best recognized in repeated follow-ups over long periods of time (fig. 3). This represents a normal feature in patients treated with conventional TACE (13).
Figure 2 - Lipiodol accumulation patterns identified in our study. a = compact; $\mathrm{b}=$ heterogeneous; $\mathbf{c}=$ ring-shaped; $d, e=$ nodular; $f=$ absent.
Figure 3 - Gradual nodule size decrease, from pre-treatment contrast enhanced arterial-phase (largest diameter $=\mathbf{3 6} \mathrm{mm}$ ) (a), to baseline $=29 \mathrm{~mm}$ (b) and four follow-up examinations, at 4 months $=21$ $\mathrm{mm}$ (c), 7 months $=16 \mathrm{~mm}$ (d),

16 months $=9 \mathrm{~mm}(\mathrm{e})$ and 26 months $=7 \mathrm{~mm}$ (f) after baseline, in a patient with no tumoral recurrence.
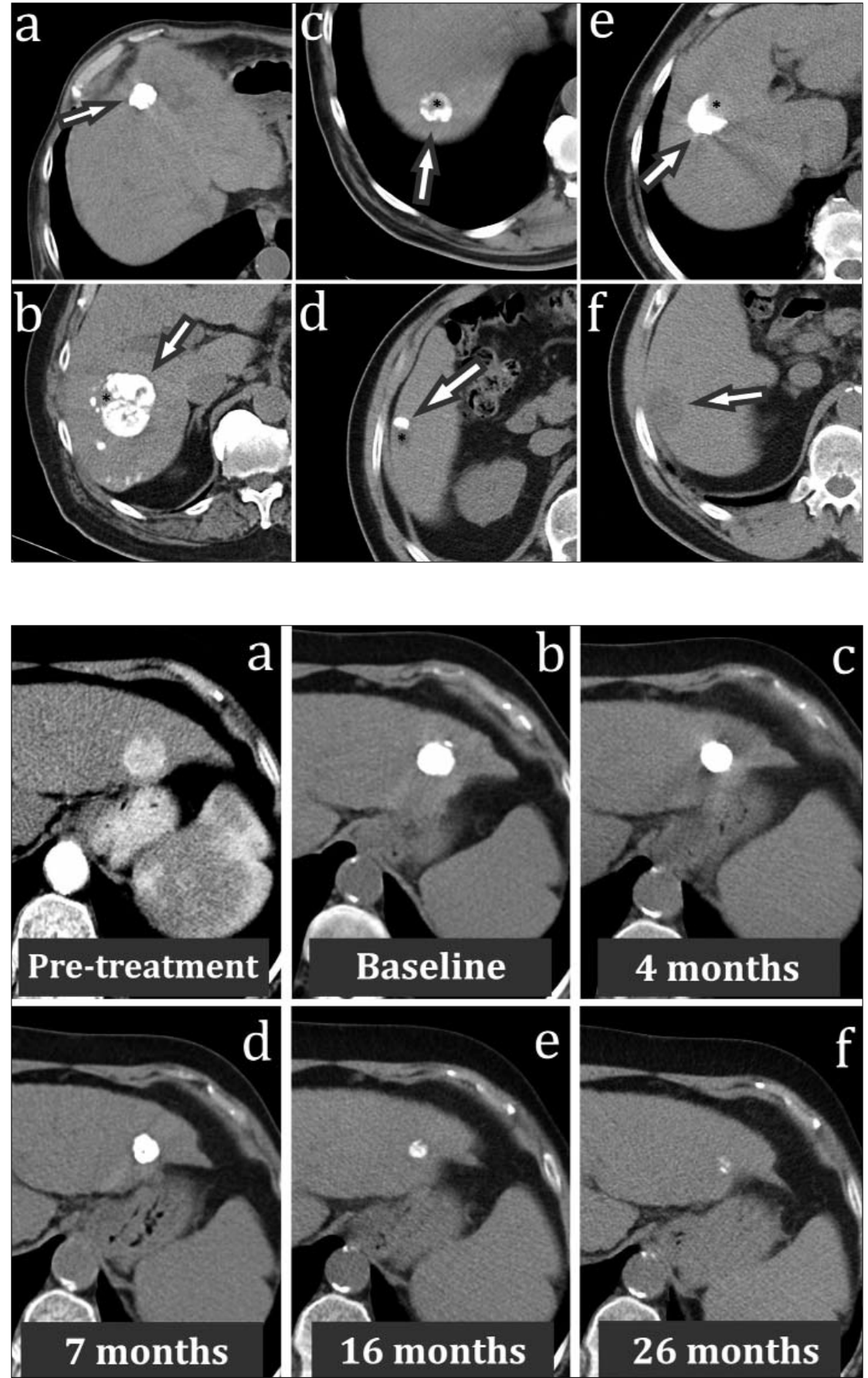
Lipiodol uptake densities, at baseline and follow-up studies, were the central focus of this paper. There was a statistically significant difference between the mean densities measured at baseline in treated tumoral nodules of patients who developed tumoral recurrence on one hand, as compared to those with no signs of recurrence at the follow-up examination on the other hand. The mean values for patients with no recurrence were $437.6 \pm 188.6 \mathrm{HU}$, compared to $289.4 \pm 289.2 \mathrm{HU}$ in patients with recurrence. The high value of the standard deviation in patients with tumoral recurrence was due to the patients with absent Lipiodol uptake $(n=2)$. No patients with absent Lipiodol accumulation pattern were free of tumoral recurrence. This information could be useful in selecting patients with high risk of developing a tumoral recurrence and employing a more strict surveillance protocol.

Furthermore, we analysed the ROC curves in order to assess the ability of baseline Lipiodol density to predict tumor recurrence. For densities $\leq 390.03 \mathrm{HU}$ of the measured ROI in the treated nodule at the baseline examination we can diagnose tumoral recurrence with a sensitivity of $84.62 \%$ and a specificity of $57.14 \%$. Although slightly higher values have been obtained in larger studies (14), these values still seem low for a diagnostic test and therefore it may appear like an inappropriate parameter to use since other methods such as contrast uptake, or Magnetic Resonance Imaging apparently yield better results (15). Yet, we argue that if we choose a cut-off value of $\leq 545.36 \mathrm{HU}$, the sensitivity for diagnosing tumoral recurrence is $92.31 \%$, albeit a specificity of only $28.57 \%$. However, considering the occurrence of a focal defect or significant washout in the Lipiodol mass is intuitively highly suggestive for viable tumoral tissue, with practically no differential diagnosis, we can sacrifice the tests' specificity in order to increase the overall positive predictive value. Consequently, this increases the sensitivity of the test by using a higher density criterion.

\section{CONCLUSIONS}

Baseline post-procedural Lipiodol accumulation levels may serve as a predictor for tumoral recurrence in patients treated for HCC using TACE, with good sensitivity provided a high cut-off value is used for the measured densities. Some post-TACE Lipiodol uptake patterns could suggest a higher chance of tumoral recurrence in patients with treated HCC. Tumoral biopsy plays a minor diagnostic role in patients with HCC in the current context of highly accurate non- invasive imaging methods. Low patient adherence to surveillance represents an impediment for early tumoral recurrence diagnostic in patients with HCC treated by TACE.

\section{REFERENCES}

1. Ryerson AB, Eheman CR, Altekruse SF, Ward JW, Jemal A, Sherman $\mathrm{RL}$, et al. Annual Report to the Nation on the Status of Cancer, 19752012, featuring the increasing incidence of liver cancer. Cancer. 2016;122(9):1312-37. doi: 10.1002/cncr.29936. Epub 2016 Mar 9.

2. McGlynn KA, London WT. The global epidemiology of hepatocellular carcinoma: present and future. Clin Liver Dis. 2011;15(2):223-43, viiX. doi: 10.1016/j.cld.2011.03.006.

3. Forner A, Reig ME, de Lope CR, Bruix J. Current strategy for staging and treatment: the BCLC update and future prospects. Semin Liver Dis. 2010;30(1):61-74. doi: 10.1055/s-0030-1247133.

4. Sun $\mathrm{H}, \mathrm{Ni}$ J, Jiang $X$, Chen D, Chen $Y, X u$ L. The effect of lipiodol deposition in HCC after TACE on the necrosis range of PMCT. Onco Targets Ther. 2017;10:3835-3842.

5. Schraml C, Kaufmann S, Rempp H, Syha R, Ketelsen D, Notohamiprodjo M, et al. Imaging of HCC-Current State of the Art. Diagnostics (Basel). 2015;5(4):513-45.

6. Vogl TJ, Schaefer P, Lehnert T, Nour-Eldin NE, Ackermann H, Mbalisike $\mathrm{E}$, et al. Intraprocedural blood volume measurement using C-arm CT as a predictor for treatment response of malignant liver tumours undergoing repetitive transarterial chemoembolization (TACE). Eur Radiol. 2016;26(3):755-63.

7. Sato $\mathrm{Y}$, Watanabe $\mathrm{H}$, Sone M, Onaya H, Sakamoto N, Osuga $\mathrm{K}$, et al. Tumor response evaluation criteria for HCC (hepatocellular carcinoma) treated using TACE (transcatheter arterial chemoembolization): RECIST (response evaluation criteria in solid tumors) version 1.1 and mRECIST (modified RECIST): JIVROSG-0602. Ups J Med Sci. 2013;118(1):16-22. doi: 10.3109/03009734.2012.729104.

8. Clinical Practice Guidelines for Hepatocellular Carcinoma Differ between Japan, United States, and Europe. Liver Cancer. 2015; 4(2):85-95. doi: 10.1159/000367730.

9. Thompson Coon J, Rogers G, Hewson P, Wright D, Anderson R, Cramp M, et al. Surveillance of cirrhosis for hepatocellular carcinoma: systematic review and economic analysis. Health Technol Assess. 2007;11(34):1-206.

10. Zhao C, Jin M, Le RH, Le MH, Chen VL, Jin M, et al. Poor adherence to hepatocellular carcinoma surveillance: a systematic review and meta-analysis of a complex issue. Liver Int. 2017 Aug 20. doi: 10.1111/liv.13555. [Epub ahead of print]

11. Franssen B, Alshebeeb K, Tabrizian P, Marti J, Pierobon ES, Lubezky $N$, et al. Differences in surgical outcomes between hepatitis B- and hepatitis C-related hepatocellular carcinoma: a retrospective analysis of a single North American center. Ann Surg. 2014;260(4):650-6; discussion 656-8.

12. Hu J, Maybody M, Cao G, Wang X, Chen H, Zhu X, et al. Lipiodol retention pattern assessed by cone beam computed tomography during conventional transarterial chemoembolization of hepatocellular carcinoma: accuracy and correlation with response. Cancer Imaging. 2016;16(1):32.

13. Herber S, Biesterfeld S, Franz U, Schneider J, Thies J, Schuchmann $\mathrm{M}$, et al. Correlation of multislice CT and histomorphology in HCC following TACE: predictors of outcome. Cardiovasc Intervent Radiol. 2008;31(4):768-77.

14. Wang X, Erinjeri JP, Jia X, Gonen M, Brown KT, Sofocleous CT, et al. Pattern of Retained Contrast on Immediate Postprocedure Computed tomography (CT) After Particle Embolization of Liver Tumors Predicts Subsequent Treatment Response. Cardiovasc Intervent Radiol. 2013;36(4):1030-8. doi: 10.1007/s00270-0120506-x. Epub 2012 Nov 14

15. Yim SY, Park BJ, Um SH, Han NY, Sung DJ, Cho SB, et al. Diagnostic performance of gadoxetic acid (Primovist)-enhanced MR imaging versus CT during hepatic arteriography and portography for small hypervascular hepatocellular carcinoma. Medicine (Baltimore). 2016;95(39):e4903. 